\title{
Localization of a Bose-Fermi mixture in a bichromatic optical lattice
}

\author{
Yongshan Cheng ${ }^{1,2, *}$ and S. K. Adhikari ${ }^{1, \dagger}$ \\ ${ }^{1}$ Instituto de Física Teórica, UNESP - Universidade Estadual Paulista, 01.140-070, São Paulo, São Paulo, Brazil \\ ${ }^{2}$ Department of Physics, Hubei Normal University, 435002 Huangshi, People's Republic of China
}

(Received 29 March 2011; published 25 August 2011)

\begin{abstract}
We study the localization of a cigar-shaped superfluid Bose-Fermi mixture in a quasiperiodic bichromatic optical lattice (OL) for interspecies attraction and intraspecies repulsion. The mixture is described by the GrossPitaevskii equation for the bosons, coupled to a hydrodynamic mean-field equation for fermions at unitarity. We confirm the existence of the symbiotic localized states in the Bose-Fermi mixture and Anderson localization of the Bose component in the interacting Bose-Fermi mixture on a bichromatic OL. The phase diagram in boson and fermion numbers showing the regions of the symbiotic and Anderson localization of the Bose component is presented. Finally, the stability of symbiotic and Anderson localized states is established under small perturbations.
\end{abstract}

DOI: 10.1103/PhysRevA.84.023632

PACS number(s): 03.75.Nt, 03.75.Lm, 64.60.Cn, 67.85.Hj

\section{INTRODUCTION}

Anderson localization of superfluid atomic gases in weak disordered potentials with a large exponential tail is currently attracting a lot of interest in both experimental and theoretical studies. In experimental studies, a disorder laser speckle [1] and a quasiperiodic optical lattice (OL) [2] have been used to localize Bose-Einstein condensates (BEC). The original description of Anderson localization was based on the localization of noninteracting quantum waves in disorder potentials due to a cancellation of wave fronts coming from different locations of the disorder potential. However, superfluid atomic gases usually are interacting and the study of Anderson localization has also been extended to the case of localization of a single-component [3,4] or binary [5] BEC under repulsive effective interactions. Both a quasiperiodic OL in one [3,6] and three dimensions [7] and a random potential [8] were used in these studies. The effect of temperature on Anderson localization has also been investigated [9].

Another topic of current interest is the problem of trapped binary superfluids, where the two components could be two different hyperfine states of the same atom $\left({ }^{87} \mathrm{Rb}\right)$ [10] or two different atoms. Experimentally, a degenerate Bose-Fermi ${ }^{87} \mathrm{Rb}^{40} \mathrm{~K}$ mixture [11], degenerate Fermi-Fermi mixture of ${ }^{40} \mathrm{~K}$ [12] and of ${ }^{6} \mathrm{Li}$ [13] were considered among others. In particular, the Feshbach resonance technique driven by a magnetic [14] or optical [15] field allows one to vary the atomic interaction that opens the possibility for the study of localization of binary condensates with controllable interactions. A Bose-Fermi mixture can exhibit quite distinct features as the number of atoms and the interspecies and intraspecies interactions are varied. For example, the intraspecies repulsive interaction combined with the interspecies attraction may give rise to the symbiotic soliton [16,17]. In Ref. [18], we demonstrated that the interspecies attraction contributes to an attractive pointlike effective potential which affects the atom density distribution and the stability properties of the condensate [19].

\footnotetext{
*yong_shan@163.com

†adhikari@ift.unesp.br; www.ift.unesp.br/users/adhikari
}

Here we investigate the symbiotic localization of a cigarshaped Bose-Fermi superfluid mixture without a trap. The Fermi superfluid is taken to be a mixture of an equal number of spin-up and spin-down components in a paired state. We also consider Anderson localization of the Bose component in the Bose-Fermi mixture in a quasiperiodic bichromatic OL when the Bose component is not localized in the absence of the OL. In both cases the intraspecies Bose interaction is taken to be repulsive and the Fermi component is considered to be at unitarity [20]. The unitarity limit is the limit of strong attraction between spin-up and spin-down fermions with the FermiFermi scattering length approaching infinity. Consequently, the properties of the Fermi superfluid in this limit become universal and independent of the Fermi-Fermi scattering length and are solely determined by the Fermi energy and Fermi momentum. The unitary limit of a Fermi superfluid can now be routinely archived and studied in a laboratory [20]. For a theoretical description of the interacting Bose-Fermi mixture, we consider the mean-field Gross-Pitaevskii (GP) equation of bosons coupled to a mean-field hydrodynamic density-functional equation [21] for fermions at unitarity. The present investigation involves numerical simulation as well as analytical study based on a variational approach. The formation of symbiotic and Anderson localizations as a function of Bose and Fermi numbers is illustrated in a phase diagram. By numerical simulation of the coupled mean-field model using the split-step Fourier spectral method, we confirm the existence of the symbiotic localized states in the Bose-Fermi mixture without external trapping potential. We also identify Anderson localization of the Bose component with an exponential tail assisted by the Fermi component in the presence of a quasiperiodic bichromatic OL. We find that the Anderson localization of the Bose component is possible in the mixture for an interspecies attraction which neutralizes mostly the bosonic repulsion. Finally, we establish the stability of both symbiotic and Anderson localizations under small perturbations.

In Sec. II we present a brief account of the coupled mean-field model and the bichromatic OL potential used in the study. The analytical expressions for the width of the Bose and Fermi localized states obtained by the variational analysis of the mean-field model are in reasonable agreement 
with the numerical solution. The numerical results for the static and stability properties of the symbiotic and Anderson states are presented in Sec. III. In Sec. IV we present a brief summary.

\section{ANALYTICAL CONSIDERATION OF LOCALIZATION}

We consider a binary superfluid Bose-Fermi mixture of $N_{\mathrm{B}}$ bosons of mass $m_{\mathrm{B}}$ and $N_{\mathrm{F}}$ fermions of mass $m_{\mathrm{F}}$ at zero temperature. The spin-half fermions are taken to be at unitarity and populated equally in spin-up and spin-down states. The intraspecies Bose interaction is taken to be repulsive and the interspecies Bose-Fermi interaction is taken to be attractive. Experimentally, this situation is, for example, accessible in a Bose-Fermi mixture [12,22] of bosonic ${ }^{87} \mathrm{Rb}$ atoms in the hyperfine state $\left|F=2, m_{\mathrm{F}}=2\right\rangle$ and fermionic ${ }^{40} \mathrm{~K}$ atoms in the two equally populated hyperfine states $\mid F=$ $\left.9 / 2, m_{\mathrm{F}}=-9 / 2\right\rangle$ and $\left|F=9 / 2, m_{\mathrm{F}}=-7 / 2\right\rangle$. Theoretically, the bosons are treated by the usual mean-field GP equation with contact interaction, which is equivalent to the standard hydrodynamical equations. The fermions are treated by a mean-field density-functional equation, which is equivalent to the standard hydrodynamical equations for the Fermi superfluid. The bosons and fermions are assumed to interact by a zero-range potential. Within the framework of the densityfunctional theory, a coupled GP-type equation for the mixture can be obtained [21,23].

The system is made effectively one-dimensional (1D), assuming that the mixture is confined in transverse directions by a tight axisymmetric harmonic potential of frequencies $\omega_{\perp \mathrm{B}}$ and $\omega_{\perp \mathrm{F}}$ for bosons and fermions, respectively. The three-dimensional equations of Ref. [21] for a cigar-shaped superfluid Bose-Fermi mixture can then be reduced to an effective 1D form by integrating out the dependence on the radial spatial variables [23]. The dynamics of the 1D mixture is described by the dimensionless coupled time-dependent nonlinear equations [23]

$$
\begin{gathered}
i \frac{\partial u_{\mathrm{B}}}{\partial t}=-\frac{1}{2} \frac{\partial^{2} u_{\mathrm{B}}}{\partial x^{2}}+g_{\mathrm{B}}\left|u_{\mathrm{B}}\right|^{2} u_{\mathrm{B}}+g_{\mathrm{BF}} N_{\mathrm{F}}\left|u_{\mathrm{F}}\right|^{2} u_{\mathrm{B}}+V(x) u_{\mathrm{B}} \\
\frac{i}{2} \frac{\partial u_{\mathrm{F}}}{\partial t}=-\frac{1}{8} \frac{\partial^{2} u_{\mathrm{F}}}{\partial x^{2}}+g_{\mathrm{F}}\left|u_{\mathrm{F}}\right|^{4 / 3} u_{\mathrm{F}}+g_{\mathrm{BF}} N_{\mathrm{B}}\left|u_{\mathrm{B}}\right|^{2} u_{\mathrm{F}}+V(x) u_{\mathrm{F}},
\end{gathered}
$$

where $V(x)$ is the trapping potential acting on both the Bose and Fermi components, $u_{\mathrm{B}} \equiv u_{\mathrm{B}}(x, t)$ and $u_{\mathrm{F}} \equiv u_{\mathrm{F}}(x, t)$ are the $1 \mathrm{D}$ wave functions with normalization $\int_{-\infty}^{\infty}\left|u_{\mathrm{B}}\right|^{2} d x=$ $\int_{-\infty}^{\infty}\left|u_{\mathrm{F}}\right|^{2} d x=1$. In Eqs. (1) and (2), we set the transverse oscillator lengths $a_{\perp \mathrm{B}}=a_{\perp \mathrm{F}} \equiv a_{\perp}$ and $\omega_{\perp \mathrm{B}}=\omega_{\perp \mathrm{F}} \equiv \omega_{\perp}$, measuring length, energy, and time in units of $a_{\perp}, \hbar \omega_{\perp}$, and $\omega_{\perp}^{-1}$, respectively [23]. This implies that $2 m_{\mathrm{F}}=m_{\mathrm{B}}$, a condition which is roughly satisfied by the ${ }^{87} \mathrm{Rb}-{ }^{40} \mathrm{~K}$ mixture. The dimensionless interactions are [23] $g_{\mathrm{B}}=2\left(a_{\mathrm{B}} / a_{\perp}\right) N_{\mathrm{B}}, g_{\mathrm{BF}}=$ $6\left(a_{\mathrm{BF}} / a_{\perp}\right), g_{\mathrm{F}}=\left(3 \pi^{2}\right)^{2 / 3}(3 \xi / 5) N_{\mathrm{F}}^{2 / 3}$, where $a_{\mathrm{B}}$ is the Bose scattering length, $a_{\mathrm{BF}}$ is the Bose-Fermi scattering length, and the universal Bertsch constant $\xi$ has the value $\xi=0.4$ at unitarity [20,24]. We consider the scattering length for collision between ${ }^{87} \mathrm{Rb}$ atoms to be positive, $a_{\mathrm{B}} \approx 108 a_{0}$ with $a_{0}$ the Bohr radius [25], whereas the interspecies scattering length between bosons $\left({ }^{87} \mathrm{Rb}\right)$ and fermions $\left({ }^{40} \mathrm{~K}\right)$ is negative, $a_{\mathrm{BF}} \approx-284 a_{0}$ [22,26]. If the Bosons are decoupled from the Fermions (by considering $a_{\mathrm{BF}}=0$ ), they satisfy the GP equation, and it was found in Ref. [4] that Anderson localization is destroyed due to Bose-Bose repulsion while the stationary states are exponentially localized [27]. Once a Bose-Fermi attraction above a critical value is introduced via a nonzero negative $a_{\mathrm{BF}}$, so that the Bose-Bose repulsion in the GP equation is nearly compensated for by interatomic attraction, localization is restored.

The coupled equations (1) and (2) will be used to study a cigar-shaped, localized Bose-Fermi system [28-30]. We study the symbiotic localized states of this system and Anderson localization of bosons in the Bose-Fermi mixture. To study the symbiotic localized states due to the strong interspecies attraction we take $V(x)=0$. To study the Anderson localization, following the experiment of Roati et al. [2], the potential $V(x)$ is taken to be a quasiperiodic bichromatic OL of incommensurate wavelengths:

$$
V(x)=\sum_{l=1}^{2} A_{l} \sin ^{2}\left(k_{l} x\right),
$$

with $A_{l}=2 \pi^{2} s_{l} / \lambda_{l}^{2}, l=1,2$, where $\lambda_{l}$ 's are the wavelengths of the OL potentials, $s_{l}$ 's are their intensities, and $k_{l}=2 \pi / \lambda_{l}$ are the corresponding wave numbers. Without losing generality, we take $s_{1}=10, s_{2}=0.3 s_{1}, \lambda_{1}=10$, and $k_{2} / k_{1}=(\sqrt{5}-$ 1)/2 [31] which roughly represent the generic experimental situation [2].

To obtain the stationary localized states of the coupled equations (1) and (2), we may set $u_{\mathrm{B}, \mathrm{F}}(x, t)=\phi_{\mathrm{B}, \mathrm{F}}(x) \exp \left(-i \mu_{\mathrm{B}, \mathrm{F}} t\right)$ with $\mu_{\mathrm{B}, \mathrm{F}}$ the respective chemical potentials. The real wave functions $\phi_{\mathrm{B}, \mathrm{F}}(x)$ obey the stationary equations

$$
\begin{aligned}
\mu_{\mathrm{B}} \phi_{\mathrm{B}} & =-\phi_{\mathrm{B}}^{\prime \prime} / 2+g_{\mathrm{B}} \phi_{\mathrm{B}}^{3}+g_{\mathrm{BF}} N_{\mathrm{F}} \phi_{\mathrm{F}}^{2} \phi_{\mathrm{B}}+V \phi_{\mathrm{B}}, \\
\mu_{\mathrm{F}} \phi_{\mathrm{F}} / 2 & =-\phi_{\mathrm{F}}^{\prime \prime} / 8+g_{\mathrm{F}} \phi_{\mathrm{F}}^{7 / 3}+g_{\mathrm{BF}} N_{\mathrm{B}} \phi_{\mathrm{B}}^{2} \phi_{\mathrm{F}}+V \phi_{\mathrm{F}},
\end{aligned}
$$

where the prime denotes space derivative. The stationary localized states can be investigated by the Gaussian variational approach [32]. In this approach, the Lagrangian density for Eqs. (4) and (5) is

$$
\begin{aligned}
\mathscr{L}= & N_{\mathrm{B}}\left[\mu_{\mathrm{B}} \phi_{\mathrm{B}}^{2}-\left(\phi_{\mathrm{B}}^{\prime}\right)^{2} / 2-g_{\mathrm{B}} \phi_{\mathrm{B}}^{4} / 2-V \phi_{\mathrm{B}}^{2}\right] \\
& +N_{\mathrm{F}}\left[\mu_{\mathrm{F}} \phi_{\mathrm{F}}^{2} / 2-\left(\phi_{\mathrm{F}}^{\prime}\right)^{2} / 8-3 g_{\mathrm{F}} \phi_{\mathrm{F}}^{10 / 3} / 5-V \phi_{\mathrm{F}}^{2}\right] \\
& -g_{\mathrm{BF}} N_{\mathrm{B}} N_{\mathrm{F}} \phi_{\mathrm{B}}^{2} \phi_{\mathrm{F}}^{2} .
\end{aligned}
$$

We use the variational trial function

$$
\phi_{\mathrm{B}, \mathrm{F}}(x)=\frac{1}{\pi^{1 / 4}} \sqrt{\frac{\mathcal{N}_{\mathrm{B}, \mathrm{F}}}{w_{\mathrm{B}, \mathrm{F}}}} \exp \left(-\frac{x^{2}}{2 w_{\mathrm{B}, \mathrm{F}}^{2}}\right),
$$

where the parameters $w_{\mathrm{B}, \mathrm{F}}$ are the widths of the Bose and Fermi localized states, respectively, with normalization $\mathcal{N}_{\mathrm{B}, \mathrm{F}}=\int_{-\infty}^{\infty} \phi_{\mathrm{B}, \mathrm{F}}^{2}(x) d x$. The trial function (7) and potential 
(3) lead to the the effective Lagrangian

$$
\begin{aligned}
L= & \int_{-\infty}^{\infty} \mathscr{L} d x-\mu_{\mathrm{B}} N_{\mathrm{B}}-\frac{1}{2} \mu_{\mathrm{F}} N_{\mathrm{F}} \\
= & \mu_{\mathrm{B}} N_{\mathrm{B}}\left(\mathcal{N}_{\mathrm{B}}-1\right)+\frac{1}{2} \mu_{\mathrm{F}} N_{\mathrm{F}}\left(\mathcal{N}_{\mathrm{F}}-1\right)-\frac{N_{\mathrm{B}} \mathcal{N}_{\mathrm{B}}}{4 w_{\mathrm{B}}^{2}} \\
& -\frac{N_{\mathrm{F}} \mathcal{N}_{\mathrm{F}}}{16 w_{\mathrm{F}}^{2}}-\frac{g_{\mathrm{B}} N_{\mathrm{B}} \mathcal{N}_{\mathrm{B}}^{2}}{2 \sqrt{2 \pi} w_{\mathrm{B}}}-\left(\frac{3}{5}\right)^{3 / 2} \frac{g_{\mathrm{F}} N_{\mathrm{F}} \mathcal{N}_{\mathrm{F}}^{5 / 3}}{\pi^{1 / 3} w_{\mathrm{F}}^{2 / 3}} \\
& -\frac{g_{\mathrm{BF}} N_{\mathrm{B}} N_{\mathrm{F}} \mathcal{N}_{\mathrm{B}} \mathcal{N}_{\mathrm{F}}}{\sqrt{\pi} \sqrt{w_{\mathrm{B}}^{2}+w_{\mathrm{F}}^{2}}}+L_{\mathrm{B}}+L_{\mathrm{F}}, \\
& L_{\mathrm{B}, \mathrm{F}}=-\frac{N_{\mathrm{B}, \mathrm{F}} \mathcal{N}_{\mathrm{B}, \mathrm{F}}}{2} \sum_{l=1}^{2} A_{l}\left(1-e^{-k_{l}^{2} w_{\mathrm{B}, \mathrm{F}}^{2}}\right) .
\end{aligned}
$$

Euler-Lagrange equations $\partial L / \partial \mu_{\mathrm{B}, \mathrm{F}}=0$ yield $\mathcal{N}_{\mathrm{B}}=\mathcal{N}_{\mathrm{F}}=$ 1. The remaining Euler-Lagrange equations $\partial L / \partial w_{\mathrm{B}, \mathrm{F}}=0$ yield, respectively,

$$
\begin{gathered}
1+\frac{g_{\mathrm{B}} w_{\mathrm{B}}}{\sqrt{2 \pi}}-2 W_{\mathrm{B}}+2 N_{\mathrm{F}} G_{\mathrm{B}}=0, \\
1+\frac{16}{3}\left(\frac{3}{5}\right)^{3 / 2} \frac{g_{\mathrm{F}} w_{\mathrm{F}}^{4 / 3}}{\pi^{1 / 3}}-8 W_{\mathrm{F}}+8 N_{\mathrm{B}} G_{\mathrm{F}}=0, \\
W_{\mathrm{B}, \mathrm{F}} \equiv w_{\mathrm{B}, \mathrm{F}}^{4} \sum_{l=1}^{2} A_{l} k_{l}^{2} e^{-k_{l}^{2} w_{\mathrm{B}, \mathrm{F}}^{2}}, \\
G_{\mathrm{B}, \mathrm{F}} \equiv \frac{g_{\mathrm{BF}} w_{\mathrm{B}, \mathrm{F}}^{4}}{\sqrt{\pi}\left(w_{\mathrm{B}}^{2}+w_{\mathrm{F}}^{2}\right)^{3 / 2}},
\end{gathered}
$$

where we have set $\mathcal{N}_{\mathrm{B}}=\mathcal{N}_{\mathrm{F}}=1$. Equations (10) and (11) give the spatial widths of the localized states.

To study how widths $w_{\mathrm{B}, \mathrm{F}}$ evolve with the particle numbers $N_{\mathrm{B}, \mathrm{F}}$, we solve Eqs. (10) and (11) and show the phase diagram of the number of bosons and fermions in Fig. 1 illustrating the regions where the localization can exist with or without a trapping potential [Eq. (3)]. The region I bounded by the solid line corresponds to $V(x)=0$ where Eqs. (10) and (11) have finite real solutions for both widths $w_{\mathrm{B}, \mathrm{F}}$. In this region, symbiotic localized states supported by interspecies BoseFermi attraction can be produced in spite of intraspecies Bose and Fermi repulsion. Generally, a self-repulsive Bose or Fermi superfluid cannot support a localized state by itself; however, a sufficiently strong interspecies attraction can induce a net effective attraction responsible for the formation of symbiotic states in a Bose-Fermi system [16].

With the bichromatic OL [Eq. (3)], Eqs. (10) and (11) yield real solutions for both widths $w_{\mathrm{B}, \mathrm{F}}$ in regions $\mathrm{I}$ and II bounded by the lines with crosses. In regions III and IV, however, finite real solutions of Eqs. (10) and (11) do not exist. In region II, the fact that the widths $w_{\mathrm{B}, \mathrm{F}}$ are finite and real implies that the localized states are created by the quasiperiodic bichromatic OL [Eq. (3)]. The parameter space in Fig. 1 where localization can exist is enlarged from region I to region II because of the bichromatic OL. The phase diagram also shows that the effect of the bichromatic OL is small when $N_{\mathrm{B}}$ is small and $N_{\mathrm{F}}>200$. For example, the region II between regions I and III is very narrow. This implies that, compared to the bichromatic OL, the interspecies

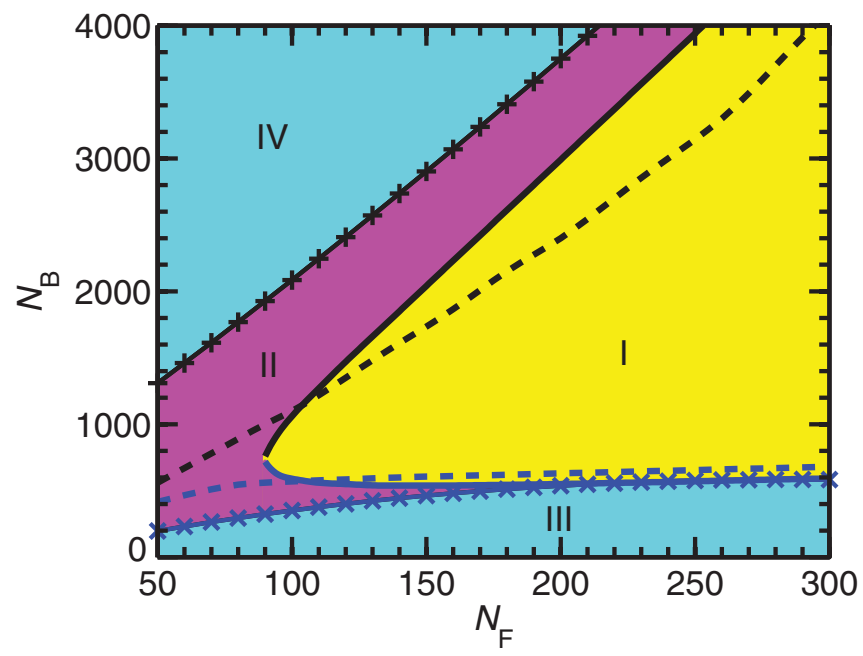

FIG. 1. (Color online) Phase diagram of Bose and Fermi atom numbers showing the regions of localization without the external trap (region I) and with the bichromatic OL [Eq. (3)] (regions I and II) from a solution of the variational equations (10) and (11). In regions III and IV the variational equations have no solution. Single-peaked localization in the bichromatic OL is possible from a numerical solution of Eqs. (1) and (2) in the region bounded by the dashed lines.

interaction is dominant in this region, because a larger $N_{\mathrm{F}}$ and small $N_{\mathrm{B}}$ induce a large enough effective interspecies attraction to localize the Bose-Fermi mixture. When $N_{\mathrm{B}}$ is larger, however, the effect of the bichromatic OL is more important on localization as indicated by a wide region II between regions I and IV.

The present discussion based on a Gaussian ansatz for the wave function has its limitation in the presence of the bichromatic OL where it is possible to have multipeak density for the components not taken care of by the simple Gaussian ansatz. Single-peaked Gaussian-type localization is possible in regions I and II. However, in the presence of the bichromatic OL, localized states can exist beyond regions I and II into regions III and IV, where at least one of the components has a multipeak density distribution along the OL. Such states are not obtainable from a Gaussian variational analysis and will be studied in Sec. III using the full numerical solution of Eqs. (1) and (2).

\section{NUMERICAL RESULTS}

We perform the numerical integration of coupled GP equations (1) and (2) employing real- and imaginary-time propagation using the split-step Fourier spectral method with space step 0.04 and time step 0.001. The time evolution is continued till convergence. We also checked the accuracy of the results by varying the space and time steps and the total number of space and time steps. Although the imaginary-time propagation method could find some of the localized states, the stability of these states was confirmed through the real-time propagation method. For studying the dynamics we used real-time propagation corresponding to the solution of the full time-dependent Eqs. (1) and (2). 

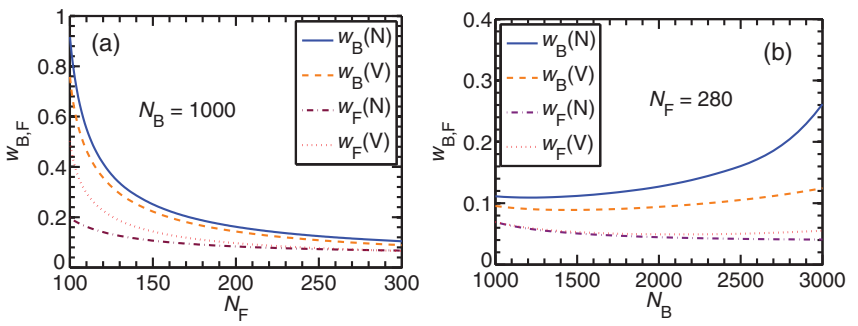

FIG. 2. (Color online) Dimensionless numerical (N) and variational $(\mathrm{V})$ widths $w_{\mathrm{B}, \mathrm{F}}$ of the Bose and Fermi components of the stationary symbiotic localized states with $V(x)=0$ vs (a) $N_{\mathrm{F}}$ for $N_{\mathrm{B}}=1000$, and vs (b) $N_{\mathrm{B}}$ for $N_{\mathrm{F}}=280$.

We first confirm the existence of the symbiotic localized states. By numerical integration of GP equations (1) and (2) with $V(x)=0$, we find that the symbiotic localized states of the Bose-Fermi systems can exist in Region I of Fig. 1. In order to investigate the effects of the number of atoms of the two components on the localized states, the typical numerical $(\mathrm{N})$ and variational $(\mathrm{V})$ widths of the atom density profiles of the stationary symbiotic localized states are exhibited in Fig. 2. The numerical widths are calculated via $w_{\mathrm{B}, \mathrm{F}}^{2}=2 \int_{-\infty}^{+\infty} x^{2}\left|u_{\mathrm{B}, \mathrm{F}}\right|^{2} d x$. We find that the numerical results are in good agreement with the variational results in the central part of region I. Near the edges (the solid line in Fig. 1), the difference between the numerical and variational widths is larger because of the deformation of the wave functions, while the atom density distributions deviate from the single-peak Gaussian shape and assume a multipeak structure. When $N_{\mathrm{B}, \mathrm{F}}$ are smaller, we find that the deformation of the fermionic component is larger than that of the bosonic component consistent with Fig. 2(a). On the contrary, the deformation of the bosonic component is larger than that of the fermionic component when $N_{\mathrm{B}, \mathrm{F}}$ are larger consistent with Fig. 2(b).

A careful analysis of the density distribution of the localized Bose-Fermi system reveals some interesting features. In fact, an attractive interspecies interaction may induce a spatially localized effective attraction [18] which can reduce the width of the atom density profiles, and even develop a sharp peak on the top of the bosonic and fermionic atom density envelopes [19] as we shall see in the following, viz., Fig. 3. Once a peak appears in the density of the first species (Bose or Fermi), this induces a highly localized effective interaction in the second species (Fermi or Bose) resulting in a highly localized structure in the second species. The small width of a species, say Bose, may, however, emerge in two ways: (i) a large overall attractive interspecies interaction $g_{\mathrm{BF}} N_{\mathrm{F}}\left|u_{\mathrm{F}}\right|^{2} u_{\mathrm{B}}$ due to a large number of the second species, $N_{\mathrm{F}}$, or (ii) a highly localized interspecies interaction $g_{\mathrm{BF}} N_{\mathrm{F}}\left|u_{\mathrm{F}}\right|^{2} u_{\mathrm{B}}$ due to a narrow localized state of the second species, Fermi.

Figure 2(a) indicates that $w_{\mathrm{B}, \mathrm{F}}$ decreases monotonically as $N_{\mathrm{F}}$ increases. This is because a larger $N_{\mathrm{F}}$ induces a larger effective attraction among bosons [16], which reduces the Bose width $w_{\mathrm{B}}$ due to possibility (i) above. Simultaneously, the narrower Bose localization causes a stronger attractive pointlike effective potential for the Fermi component, which reduces the Fermi width $w_{\mathrm{F}}$ due to possibility (ii). Figure 2(b)
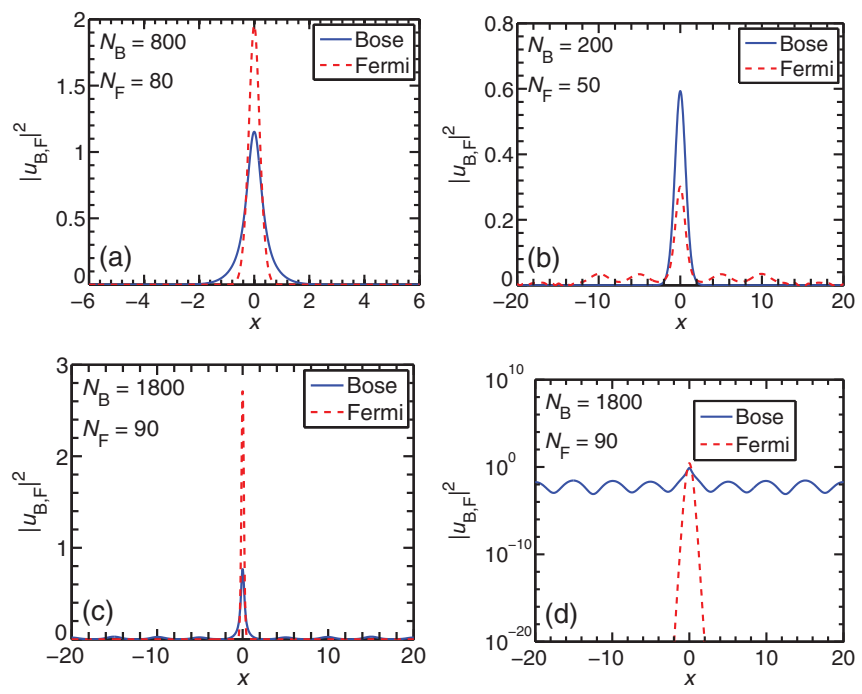

FIG. 3. (Color online) Numerical densities $\left|u_{\mathrm{B}, \mathrm{F}}\right|^{2}$ of the coupled Bose-Fermi localized states with the bichromatic OL [Eq. (3)] vs dimensionless position $x$ for (a) $N_{\mathrm{B}}=800, N_{\mathrm{F}}=80$, (b) $N_{\mathrm{B}}=$ $200, N_{\mathrm{F}}=50$, (c) $N_{\mathrm{B}}=1800, N_{\mathrm{F}}=90$, and (d) $N_{\mathrm{B}}=1800, N_{\mathrm{F}}=90$ in logarithmic scale. The solid line corresponds to bosons and the dashed line to fermions.

shows that, as $N_{\mathrm{B}}$ increases, $w_{\mathrm{F}}$ decreases and $w_{\mathrm{B}}$ increases. With the increase of $N_{\mathrm{B}}$ for a fixed $N_{\mathrm{F}}$, the Bose species becomes more repulsive due to an increase of intraspecies repulsion for a roughly fixed interspecies attraction, thus increasing the width of the Bose species. However, the increase of $N_{\mathrm{B}}$ for a fixed $N_{\mathrm{F}}$ increases the interspecies attraction on the Fermi species, thus reducing the Fermi width.

Next, we study the Bose-Fermi localized states with the quasiperiodic OL [Eq. (3)] in region II of Fig. 1. Typical atom density profiles of stationary Bose and Fermi localized states are shown in Figs. 3(a)-3(d). We find that, because of the bichromatic OL, the localized Bose or Fermi states may exist beyond region I. For example, Fig. 3(a) shows both Bose and Fermi components in single-peaked configuration for parameters corresponding to region II. The numerical simulation indicates, however, that both components can be single peaked only in the region between the dashed lines in Fig. 1. We name this region the single-fragment region. In the region below the single-fragment region (viz., the region with smaller $N_{\mathrm{B}}$ ), the atom density profile of the Bose component is single peaked, but the Fermi component shows a symmetrical multipeak structure with a pronounced peak at the center, as shown in Fig. 3(b). However, in the region above the single-fragment region (viz., the region with larger $N_{\mathrm{B}}$ ), the density profile of the Fermi component is single peaked and that of the Bose component is multipeaked as shown in Figs. 3(c) and 3(d). To show this clearly, Fig. 3(d) is plotted in a logarithmic scale which shows that the Bose density profile develops undulating tails occupying many OL sites. When $N_{\mathrm{B}}$ is larger, as in Figs. 3(c) and 3(d), the Bose system becomes more repulsive and hence spreads to many OL sites and thus develops undulating tails. However, a large $N_{\mathrm{B}}$ also introduces a strong interspecies attraction on the Fermi component, thus confining it in a small region. This narrow Fermi peak creates a smaller narrow peak in the Bose 

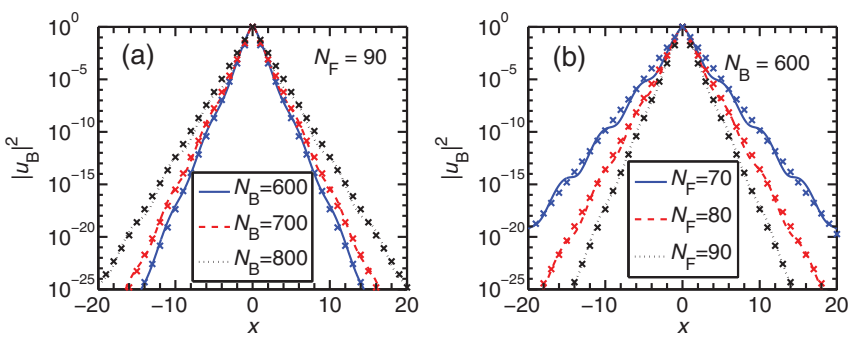

FIG. 4. (Color online) Numerical density $\left|u_{\mathrm{B}}\right|^{2}$ of the Bose component in the Bose-Fermi mixture on the bichromatic OL [Eq. (3)] vs dimensionless $x$ in logarithmic scale for (a) $N_{\mathrm{F}}=90$ and $N_{\mathrm{B}}=600$ (solid line), 700 (dashed line), 800 (dotted line), and for (b) $N_{\mathrm{B}}=600$ and $N_{\mathrm{F}}=70$ (solid line), 80 (dashed line), 90 (dotted line). The crosses are exponentially fitted to density tails $\sim \exp \left(-|x| / L_{\mathrm{loc}}\right)$, where $L_{\mathrm{loc}}$ is the dimensionless localization length.

component due to interspecies attraction as seen in Fig. 3(c). The role of Bose and Fermi is interchanged for a small $N_{\mathrm{B}}$ as can be seen in Fig. 3(b), where one has a single-peak Bose distribution on top of a multipeak Fermi distribution. The situation for an intermediate value of $N_{\mathrm{B}}$ is shown in Fig. 3(a).

Now we consider the possibility of Anderson localization of the bosons with an exponential tail in density in the interacting Bose-Fermi mixture on the bichromatic OL [Eq. (3)] [1-3,6,31]. There are domains around region II of the phase diagram shown in Fig. 1 where the interand intra-species interaction on the bosons approximately "cancel" each other. Consequently, the bosons behave like quasifree particles (with effective weak repulsion) and the weak quasiperiodic bichromatic OL is necessary for localizing the bosons. In order to understand the novel phenomenon, we investigate the localization of Bose and Fermi components in the bichromatic OL [Eq. (3)] away from the domain of strong interspecies interactions, where there is no localization in the absence of the bichromatic OL potential, e.g., around region II of Fig. 1. In that case, we find that the wave function of the Bose component may possess a pronounced exponential tail demonstrating Anderson localization. However, we could not find a similar exponential tail of fermion density distribution in the whole phase diagram in Fig. 1.

To study Anderson localization of the Bose component, while both Bose and Fermi components are single peaked under the action of the OL trap [Eq. (3)], we plot in Fig. 4 the atom density $\left|u_{\mathrm{B}}\right|^{2}$, in log scale, of the stationary Bose localized states. The central part of the atom density is quasi-Gaussian, however, with a long exponential tail. We have also shown in Fig. 4 the exponential fitting to density tails with $\sim \exp \left(-|x| / L_{\text {loc }}\right)$, where $L_{\text {loc }}$ is the localization length. (Note that our definition of localization length [3] differs from that of Refs. $[1,6]$ by a factor of 2.) The length $L_{\text {loc }}$ depends on the effective nonlinearity of the bosonic component and the disorder of the quasiperiodic OL [33]. Here, we fix the quasiperiodic OL [Eq. (3)] and study the effect of nonlinearity on localization length. In Fig. 4(a), for $N_{\mathrm{F}}=90, L_{\text {loc }}=0.25$ for $N_{\mathrm{B}}=600, L_{\mathrm{loc}}=0.28$ for $N_{\mathrm{B}}=700$, and $L_{\mathrm{loc}}=0.35$ for $N_{\mathrm{B}}=800$ and the localization length increases with $N_{\mathrm{B}}$. This is because that a larger $N_{\mathrm{B}}$, for a fixed $N_{\mathrm{F}}$, implies a larger repulsive bosonic nonlinearity, thus resulting in a larger
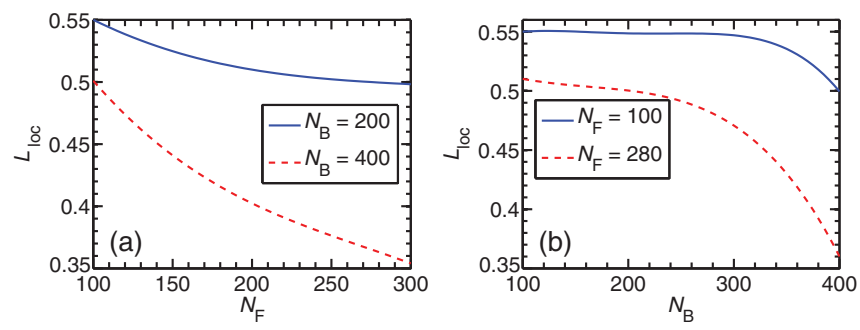

FIG. 5. (Color online) Dimensionless localization length $L_{\text {loc }}$ of Bose density $\left|u_{\mathrm{B}}\right|^{2}$ vs (a) $N_{\mathrm{F}}$ for $N_{\mathrm{B}}=200,400$, and vs (b) $N_{\mathrm{B}}$ for $N_{\mathrm{F}}=100,280$, in the Bose-Fermi mixture on the bichromatic OL [Eq. (3)].

value of localization length. Figure 4(b) indicates that, for a fixed $N_{\mathrm{B}}=600, L_{\mathrm{loc}}$ decreases with $N_{\mathrm{F}}$ with $L_{\mathrm{loc}}=0.44$ for $N_{\mathrm{F}}=70, L_{\mathrm{loc}}=0.32$ for $N_{\mathrm{F}}=80$, and $L_{\mathrm{loc}}=0.25$ for $N_{\mathrm{F}}=90$. A larger $N_{\mathrm{F}}$, for a fixed $N_{\mathrm{B}}$, implies a larger interspecies attraction on the bosonic component [16], thus reducing the localization length.

We also investigate numerically the Anderson localization of the Bose component [Eq. (3)] for small $N_{\mathrm{B}}$. With the OL [Eq. (3)], the Bose localization length $L_{\text {loc }}$ vs $N_{\mathrm{F}}$ and $N_{\mathrm{B}}$ is illustrated in Figs. 5(a) and 5(b), respectively. As shown in Fig. 5(a), because of interspecies attraction, $L_{\text {loc }}$ for a fixed $N_{\mathrm{B}}$ monotonically decreases with the increase of $N_{\mathrm{F}}$. For smaller $N_{\mathrm{B}}(=200)$, there is a saturation of $L_{\mathrm{loc}}$ and Anderson localization with an exponential tail continues with the increase of $N_{\mathrm{F}}$. For larger $N_{\mathrm{B}}(=400), L_{\mathrm{loc}}$ is reduced rapidly with the increase of $N_{\mathrm{F}}$ until the Anderson localization with an exponential tail is destroyed, and one has strong localization without an exponential tail. In Fig. 5(b), we show the variation of $L_{\mathrm{loc}}$ with $N_{\mathrm{B}}$ for $N_{\mathrm{F}}=100$ and 280 . From the phase plot in Fig. 1 we find that in both cases Anderson localization is rapidly destroyed as we enter the region I of Fig. 1 of permanent symbiotic trapping with the increase of $N_{\mathrm{B}}$, and the localization length is reduced rapidly.

Now we study numerically some dynamics of the localized Bose-Fermi states and test their stability. First, we consider a Bose-Fermi state in the region I of Fig. 1 corresponding to permanent symbiotic trapping. To do this, first we create the stationary localized Bose-Fermi mixture under appropriate conditions. Successively, at $t=0$, we suddenly introduce a phase $\exp \left(i p_{\mathrm{B}, \mathrm{F}} x\right)$ in the wave function of one of the components to initiate a translational motion. No momentum is given to the second component. By introducing an initial translation to the Bose or Fermi component, the evolution of the atom density envelopes is presented in Fig. 6 for $N_{\mathrm{B}}=1000, N_{\mathrm{F}}=160$, and for (a) $p_{\mathrm{B}}=0.02, p_{\mathrm{F}}=0$, and for (b) $p_{\mathrm{B}}=0, p_{\mathrm{F}}=0.1$. We find that the two components remain bound together and move with the same constant velocity although the initial momentum is given to only one component. The binding is caused by the stronger interspecies attraction. We see in Fig. 6 that the symbiotic Bose-Fermi locazed states remain unchanged after perturbation, which confirms the stability of these states.

Next we consider the dynamics of an Anderson localized Bose state in the Bose-Fermi mixture on the bichromatic OL [Eq. (3)] with $N_{\mathrm{B}}=800$ and $N_{\mathrm{F}}=90$ corresponding to one of the localized states of Fig. 4(a). The dynamics is initiated by 

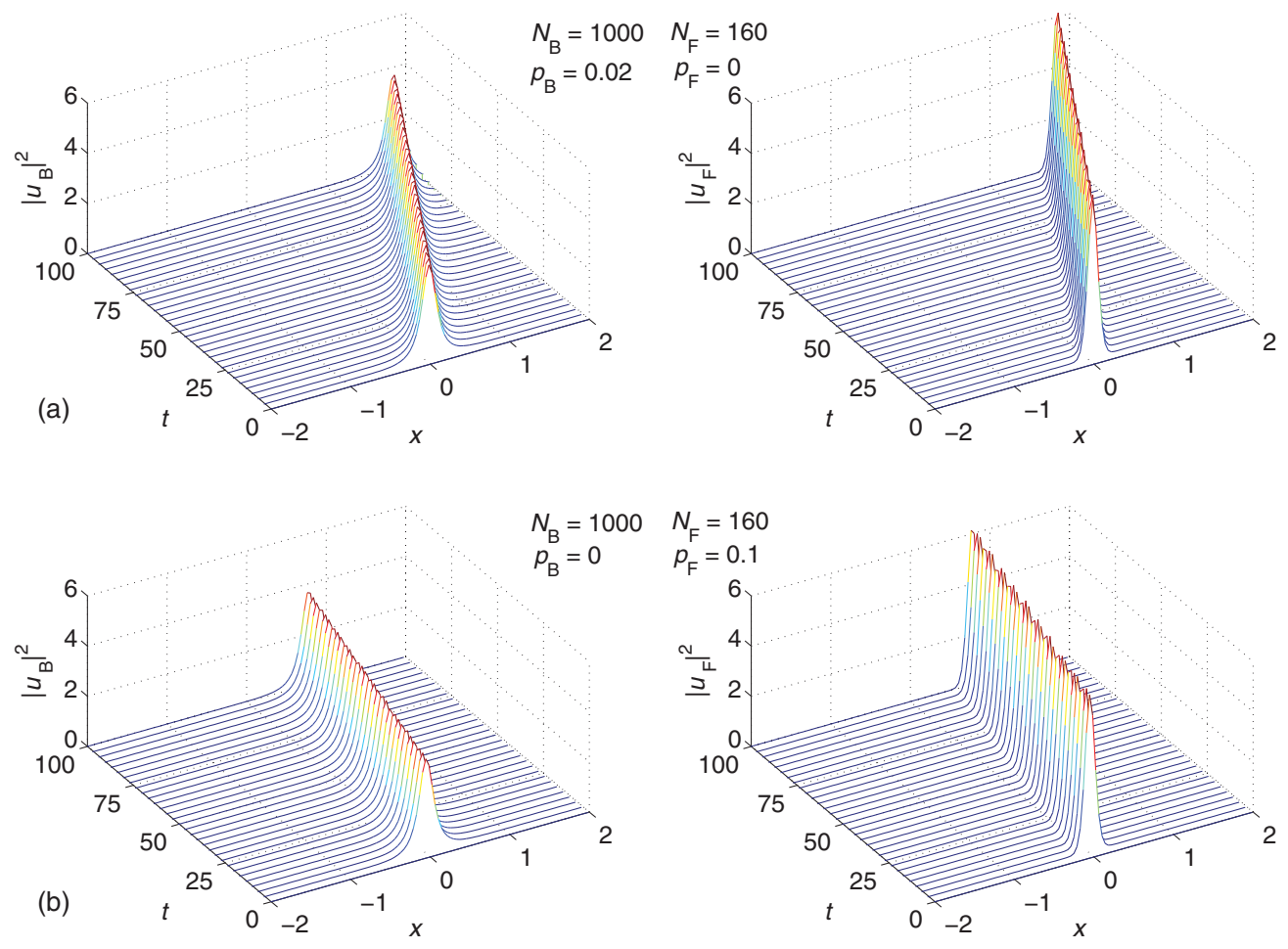

FIG. 6. (Color online) Numerical densities $\left|u_{\mathrm{B}, \mathrm{F}}\right|^{2}$ vs dimensionless $x$ and $t$ of the symbiotic Bose-Fermi localized states after introducing a momentum in one of the components by transformation $u_{\mathrm{B}, \mathrm{F}} \rightarrow u_{\mathrm{B}, \mathrm{F}} \exp \left(i p_{\mathrm{B}, \mathrm{F}} x\right)$. The parameters are $N_{\mathrm{B}}=1000, N_{\mathrm{F}}=160$ and $(\mathrm{a}) p_{\mathrm{B}}=0.02$, $p_{\mathrm{F}}=0$, and (b) $p_{\mathrm{B}}=0, p_{\mathrm{F}}=0.1$.

introducing a phase $\exp \left(i p_{\mathrm{B}, \mathrm{F}} x\right)$ in the wave function of one of the components to start a translational motion. Because of the quasiperiodic OL [Eq. (3)], the bound Bose-Fermi localized state oscillates together periodically about the origin in one site of the OL after introducing an initial momentum into one of the components, as shown in Fig. 7 which shows only the movement of the center of the Bose component, e.g.,

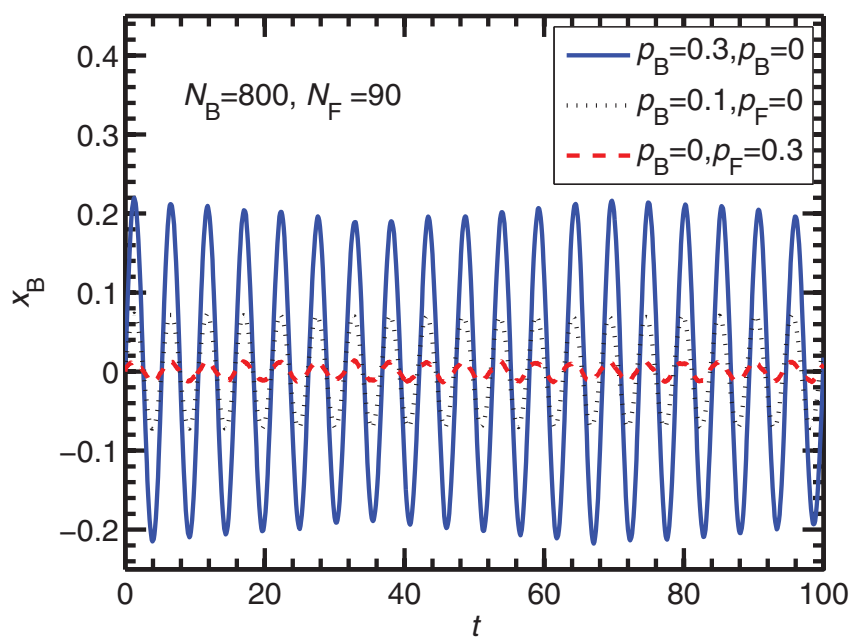

FIG. 7. (Color online) Dimensionless center of the Bose state $x_{\mathrm{B}}$ vs dimensionless time $t$ during the location oscillation of the BoseFermi mixture on the bichromatic OL [Eq. (3)] initiated suddenly by introducing an initial momentum $p_{\mathrm{B}, \mathrm{F}}$ through the transformation $u_{\mathrm{B}, \mathrm{F}} \rightarrow u_{\mathrm{B}, \mathrm{F}} \exp \left(i p_{\mathrm{B}, \mathrm{F}} x\right)$.
$x_{\mathrm{B}}$ vs $t$. Different initial momenta result in oscillations with different amplitudes [see the solid line $\left(p_{\mathrm{B}}=0.3\right)$, the dashed line $\left(p_{\mathrm{F}}=0.3\right)$, and the dotted line $\left(p_{\mathrm{B}}=0.1\right)$ in Fig. 7]. Sustained oscillation of the Bose (as well as the bound Fermi component not shown here) component confirms the stability of the coupled Bose-Fermi state.

\section{SUMMARY}

Here we studied symbiotic localization in a cigar-shaped Bose-Fermi superfluid with intraspecies repulsion and interspecies attraction. The Fermi component is considered at unitarity. In the presence of a quasiperiodic bichromatic OL, the Bose component in the Bose-Fermi mixture could exhibit Anderson localization with a large exponential tail due to a near cancellation of the intraspecies repulsion and interspecies attraction while no localization is possible in the absence of the OL. No evidence of Anderson localization with a long exponential tail was found in the Fermi component. The cigar-shaped Bose-Fermi mixture is described by an effective one-dimensional GP equation for bosons coupled to a mean-field hydrodynamic equation for fermions at unitarity [23]. In this study, we use both numerical and variational solutions of the mean-field equation for the mixture. We obtain a phase plot of the number of bosonic and fermionic atoms showing the domain of symbiotic localization without the external trap and localization in the presence of a quasiperiodic bichromatic OL. The numerical and variational densities of the localized states are in good agreement with each other. Both symbiotic localization and Anderson localization 
are found to be dynamically stable when given a small initial velocity to one of the components. The Bose and Fermi components are found to move together under such a perturbation. With present know-how, it should be possible to study the Anderson localization of the Bose component in the Bose-Fermi mixture on a bichromatic OL experimentally under appropriate conditions.

\section{ACKNOWLEDGMENTS}

FAPESP (Brazil) and CNPq (Brazil) provided partial support. The Science and Technology Program of the Education Department of Hubei, China, under Grants Nos. D200722003 and Z200722001, provided support during the initial stage of the project in China.
[1] J. Billy, V. Josse1, Z. Zuo et al., Nature (London) 453, 891 (2008).

[2] G. Roati, C. D'Errico, and L. Fallani et al., Nature (London) 453, 895 (2008).

[3] Y. Cheng and S. K. Adhikari, Phys. Rev. A 83, 023620 (2011); 81, 023620 (2010); Laser Phys. Lett. 7, 824 (2010).

[4] A. S. Pikovsky and D. L. Shepelyansky, Phys. Rev. Lett. 100, 094101 (2008); S. Flach, D. O. Krimer, and Ch. Skokos, ibid. 102, 024101 (2009); I. García-Mata and D. L. Shepelyansky, Phys. Rev. E 79, 026205 (2009); Ch. Skokos, D. O. Krimer, S. Komineas, and S. Flach, ibid. 79, 056211 (2009); P. Lugan, D. Clement, P. Bouyer, A. Aspect, and L. Sanchez-Palencia, Phys. Rev. Lett. 99, 180402 (2007); J. E. Lye, L. Fallani, C. Fort, V. Guarrera, M. Modugno, D. S. Wiersma, and M. Inguscio, Phys. Rev. A 75, 061603(R) (2007).

[5] Y. Cheng and S. K. Adhikari, Phys. Rev. A 81, 023620 (2010).

[6] S. K. Adhikari and L. Salasnich, Phys. Rev. A 80, 023606 (2009).

[7] S. K. Adhikari, Phys. Rev. A 81, 043636 (2010).

[8] Y. Cheng and S. K. Adhikari, Phys. Rev. A 82, 013631 (2010); L. Sanchez-Palencia, D. Clement, P. Lugan, P. Bouyer, G. V. Shlyapnikov, and A. Aspect, Phys. Rev. Lett. 98, 210401 (2007); D. Clément, A. F. Varon, M. Hugbart, J. A. Retter, P. Bouyer, L. Sanchez-Palencia, D. M. Gangardt, G. V. Shlyapnikov, and A. Aspect, ibid. 95, 170409 (2005); J. E. Lye, L. Fallani, M. Modugno, D. S. Wiersma, C. Fort, and M. Inguscio, ibid. 95, 070401 (2005); B. Damski, J. Zakrzewski, L. Santos, P. Zoller, and M. Lewenstein, ibid. 91, 080403 (2003); T. Schulte, S. Drenkelforth, J. Kruse, W. Ertmer, J. Arlt, K. Sacha, J. Zakrzewski, and M. Lewenstein, ibid. 95, 170411 (2005); G. Srinivasan, A. Aceves, and D. M. Tartakovsky, Phys. Rev. A 77, 063806 (2008).

[9] I. L. Aleiner, B. L. Altshuler, and G. V. Shlyapnikov, Nat. Phys. 6, 900 (2010); D. M. Basko, I. L. Aleiner, and B. L. Altshuler, Phys. Rev. B 76, 052203 (2007).

[10] C. J. Myatt, E. A. Burt, R. W. Ghrist, E. A. Cornell, and C. E. Wieman, Phys. Rev. Lett. 78, 586 (1997); J. Stenger, S. Inouye, D. M. Stamper-Kurn et al., Nature (London) 396, 345 (1998).

[11] G. Modugno, G. Ferrari, and G. Roati et al., Science 294, 1320 (2001).

[12] B. DeMarco and D. S. Jin, Science 285, 1703 (1999).

[13] K. E. Strecker, G. B. Partridge, and R. G. Hulet, Phys. Rev. Lett. 91, 080406 (2003); G. B. Partridge, W. Li, Y. A. Liao, R. G. Hulet, M. Haque, and H. T. C. Stoof, ibid. 97, 190407 (2006).

[14] S. Inouye, M. R. Andrews, J. Stenger, H.-J. Miesner, D. M. Stamper-Kurn, and W. Ketterle, Nature (London) 392, 151 (1998).

[15] M. Theis, G. Thalhammer, K. Winkler, M. Hellwig, G. Ruff, R. Grimm, and J. H. Denschlag, Phys. Rev. Lett. 93, 123001 (2004).
[16] S. K. Adhikari, Phys. Lett. A 346, 179 (2005); Phys. Rev. A 72, 053608 (2005); J. Phys. A 40, 2673 (2007); S. K. Adhikari and L. Salasnich, Phys. Rev. A 76, 023612 (2007); L. Salasnich, S. K. Adhikari, and F. Toigo, ibid. 75, 023616 (2007).

[17] V. M. Pérez-García and J. B. Beitia, Phys. Rev. A 72, 033620 (2005).

[18] Y. S. Cheng, J. Phys. B 42, 205005 (2009); Y. S. Cheng, R. Z. Gong, and H. Li, Opt. Express 14, 3594 (2006).

[19] B. T. Seaman, L. D. Carr, and M. J. Holland, Phys. Rev. A 71, 033609 (2005); D. J. Frantzeskakis, G. Theocharis, F. K. Diakonos, P. Schmelcher, and Y. S. Kivshar, ibid. 66, 053608 (2002).

[20] L. Luo and J. E. Thomas, J. Low Temp. Phys. 154, 1 (2009).

[21] S. K. Adhikari and L. Salasnich, Phys. Rev. A 78, 043616 (2008); S. K. Adhikari, ibid. 79, 023611 (2009); Laser Phys. Lett. 6, 901 (2009); J. Phys. B 43, 085304 (2010).

[22] G. Modugno et al., Science 297, 2240 (2002); K. Günter, T. Stoferle, H. Moritz, M. Kohl, and T. Esslinger, Phys. Rev. Lett. 96, 180402 (2006); Th. Best, S. Will, U. Schneider, L. Hackermuller, D. vanOosten, I. Bloch, and D. S. Luhmann, ibid. 102, 030408 (2009).

[23] S. K. Adhikari, B. A. Malomed, L. Salasnich, and F. Toigo, Phys. Rev. A 81, 053630 (2010).

[24] S. Giorgini, L. P. Pitaevskii, and S. Stringari, Rev. Mod. Phys. 80, 1215 (2008).

[25] F. Dalfovo, S. Giorgini, L. P. Pitaevskii, and S. Stringari, Rev. Mod. Phys. 71, 463 (1999).

[26] G. Roati, F. Riboli, G. Modugno, and M. Inguscio, Phys. Rev. Lett. 89, 150403 (2002).

[27] C. Albanese and J. Fröhlich, Commun. Math. Phys. 116, 475 (1988); C. Albanese, J. Fröhlich, and T. Spencer, ibid. 119, 677 (1988); C. Albanese and J. Fröhlich, ibid. 138, 193 (1991).

[28] W. Yi and L.-M. Duan, Europhys. Lett. 75, 854 (2006).

[29] A. M. Belemuk, N. M. Chtchelkatchev, V. N. Ryzhov, and S. T. Chui, Phys. Rev. A 73, 053608 (2006).

[30] S. K. Adhikari and B. A. Malomed, Phys. Rev. A 76, 043626 (2007).

[31] M. Modugno, New J. Phys. 11, 033023 (2009); M. Larcher, F. Dalfovo, and M. Modugno, Phys. Rev. A 80, 053606 (2009); M. Larcher, M. Modugno, and F. Dalfovo, ibid. 83, 013624 (2011).

[32] V. M. Pérez-García, H. Michinel, J. I. Cirac, M. Lewenstein, and P. Zoller, Phys. Rev. A 56, 1424 (1997); S. K. Adhikari and B. A. Malomed, ibid. 79, 015602 (2009); B. A. Malomed, Prog. Opt. 43, 71 (2002).

[33] L. Sanchez-Palencia, D. Clément, P. Lugan, P. Bouyer, and A. Aspect, New J. Phys. 10, 045019 (2008); L. Fallani, C. Fort, and M. Inguscio, Adv. At. Mol. Opt. Phys. 56, 119 (2008). 